\title{
CRISPR Del/Rei: A simple, flexible and efficient pipeline for scarless genome editing
}

\author{
Marah H. Wahbeh¹,2, Kyra L. Feuer ${ }^{* 1,2}$, Sara Abdollahi ${ }^{1}$, Christian Yovo ${ }^{1}$, Eman Rabie ${ }^{3,4}$, Anh-Thu N. \\ Lam $^{1}$, Lindsay J. Young ${ }^{1}$, Dimitrios Avramopoulos ${ }^{1}$
}

${ }^{1}$ McKusick-Nathans Department of Genetic Medicine, Johns Hopkins School of Medicine, Baltimore, USA. ${ }^{2}$ Predoctoral Training Program in Human Genetics and Molecular Biology, Johns Hopkins School of Medicine, Baltimore, USA. ${ }^{3}$ Medical Molecular Genetics Department, Human Genetics and Genome Research

Division, National Research Centre, Cairo, Egypt. ${ }^{4}$ Biotechnology Program, School of Sciences and Engineering, The American University in Cairo, Cairo, Egypt.

*These authors contributed equally to this work. Correspondence should be addressed to: Dimitrios Avramopoulos (adimitr1@jhmi.edu).

Submitted to bioRxiv.org on January $18^{\text {th }}, 2021$.

Scarless genome editing is an important tool for the accurate recapitulation of genetic variation in human disease models. Various CRISPR/Cas9-based scarless editing methods have been reported. However, some of these methods have low editing efficiency (1-5\%) and require manual selection of hundreds of clones to reach the desired number. Other protocols use large selection cassettes with laborious vector assembly and specialized reagents and equipment, or have poorly understood off-target effects. To address these limitations, we developed a simple, highly efficient scarless editing strategy to edit DNA sequences in induced pluripotent stem cells, which we call CRISPR Del/Rei. This novel editing strategy consists of a two-step deletion-reinsertion strategy that produces isogenic clones in $\sim 8$ weeks using accessible, user-friendly reagents. The editing efficiency ranges from $\sim 15-100 \%$ for Step 1 and $\sim 5-20 \%$ for Step 2 after selection, which greatly reduces the amount of required manual clone isolation. Screening the transfected bulk cells and the individual clones is rapid and simple, consisting of PCR and gel electrophoresis. Despite the two editing steps, off-target effects are rare. Additionally, the experiment is well-controlled because the same protocol generates isogenic clones carrying all variant alleles. In this way, CRISPR Del/Rei serves as a valuable addition to the evolving CRISPR/Cas9 gene-editing toolset.

\section{KEYWORDS}

CRISPR/Cas9, genome editing, scarless, footprint-free, stem cells, induced pluripotent stem cells

\section{INTRODUCTION}

The CRISPR/Cas9 system for disease modeling

The CRISPR/Cas9 genome editing system originates from Clustered Regularly Interspaced Short Palindromic Repeats (CRISPR) in bacteria and archaea. In these species, CRISPR plays a role in immunity against invading phages, plasmids, and other mobile genetic elements ${ }^{1 ; 2}$. Small RNAs transcribed from CRISPR sequences are coupled with the Cas9 endonuclease to precisely target and cleave specific sequences of foreign nucleic acids. The adapted CRISPR/Cas9 editing system (Fig. 1a) consists of a Cas9 nuclease and a single guide RNA (sgRNA) with a $20 \mathrm{bp}$ spacer sequence. The spacer is complementary to a target site in the genome (the protospacer) and recruits Cas9 to create a site-specific Double-Stranded Break (DSB) in the DNA ${ }^{3}$. The most commonly used Cas9 species is $S$. pyogenes and it requires the presence of a protospacer-adjacent motif (PAM) of the sequence 'NGG' to be present immediately downstream of the protospacer ${ }^{1}$. The resulting DSB activates the cellular repair machinery which consists of two main pathways: non-homologous end joining (NHEJ, Fig. 1b), and homology-directed repair (HDR, Fig. 1c-d) ${ }^{4}$. NHEJ characteristically results in the formation of nonspecific insertions and deletions (indels) and thus can be leveraged for the efficient knock-out of genes or genetic elements. Conversely, HDR uses a homologous DNA repair template to precisely correct the DSB through recombination. This allows for the introduction of specific mutations into the genome via the repair template and is thus suited for developing models of disease-associated genetic variation. However, HDR is much less efficient than NHEJ in mammalian cells ${ }^{4}$, especially in stem cells ${ }^{5-8}$.

In the last decade, CRISPR/Cas9 technology has rapidly advanced and produced increasingly precise and diverse genome editing tools. Such tools are extremely useful for studying the consequences of genetic variation, creating human disease models, and exploring a wide array of biological questions. A particularly powerful application of the CRISPR/Cas9 system is the creation of isogenic human stem cell lines (e.g., induced pluripotent stem cells (iPSCs)) with disease-associated variation and differentiation of these lines into appropriate cell types. This approach allows for precise analysis of the implications of such variation in relevant biological contexts. 


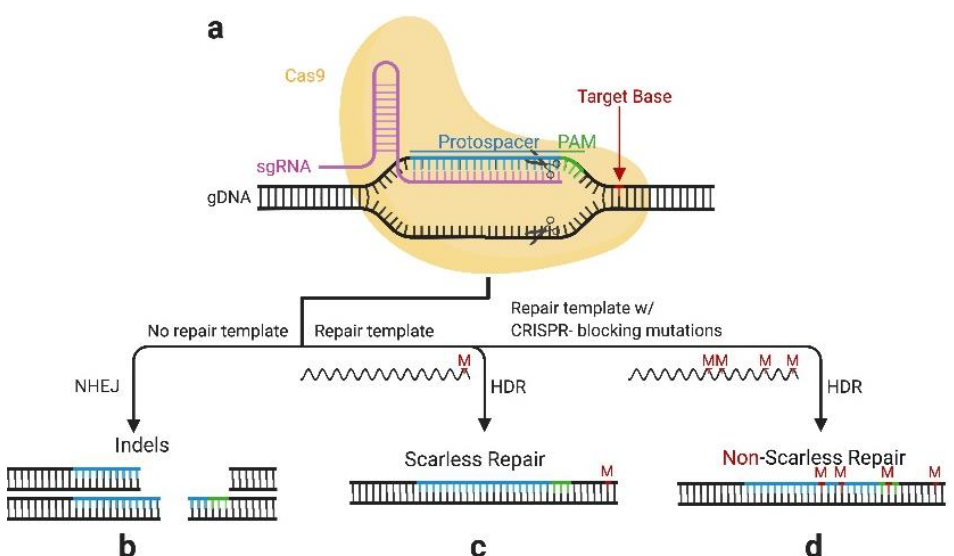

Figure 1 | The CRISPR/Cas9 editing system and repair mechanisms. (a) A user-designed 20bp single guide RNA (sgRNA) binds its complementary protospacer sequence in the genomic DNA (gDNA) close to the target base to be edited. The sgRNA recruits Cas9, which recognizes the protospacer adjacent motif (PAM) site and makes a double stranded break (DSB) in the gDNA 3bp upstream of the PAM site. (b) In the absence of a repair template the DSB is repaired by Non-Homologous End Joining (NHEJ), which results in the formation of nonspecific insertions and deletions (indels). (c-d) If a repair template is present, the DSB is repaired by Homology-Directed Repair (HDR). (c) Scarless repair occurs when a variant is introduced via the repair template at the target base without any additional mutations. (d) Non-scarless repair occurs when additional mutations are introduced; usually these are "CRISPR-blocking" and disrupt the PAM and/or protospacer to increase editing efficiency.

\section{The relevance and challenge of scarless editing}

When using CRISPR/Cas9 to develop models

of disease-associated variation, it is ideal to introduce variants of interest "scarlessly" or "footprint-free". Scarless editing is the introduction of a genetic variant without any additional mutations (Fig. 1C). This precision is important for disease modeling because the addition of extra mutations may confound the effects of the disease-associated variant. For coding regions, non-synonymous mutations may change the structure and function of the protein product; and synonymous mutations can still affect splicing ${ }^{9}$, mRNA stability ${ }^{10}$, and even substrate specificity ${ }^{11}$ of coded proteins. Likewise, the genetic code underlying noncoding regulatory sequences is not well understood and adding extra mutations in non-coding regions can have unpredictable consequences. Additionally, the increasing appreciation of the role of non-coding regulatory variation in both common ${ }^{12 ; 13}$ and rare 14-17 diseases further stresses the need for methods that allow for scarless editing.

However, scarless editing is challenging. Unless the introduced variant lies in the protospacer or the PAM site, Cas9 will be repeatedly recruited to cut its target site. This continual cleavage inevitably results in NHEJ, leading to a high percentage of indels and substantially reducing HDR efficiency ${ }^{18}$. A common approach to resolve this issue is to introduce additional "CRISPR-blocking" mutations (Fig. 1d) that disrupt the PAM site and/or protospacer to prevent subsequent
Cas9 recruitment (some refer to this as scarless editing because of the resulting precise edit without indel formation ${ }^{18}$ ). This approach is particularly common if the target base is in coding space, where the CRISPRblocking mutations are designed to be synonymous and assumed to have no effects on the protein. However, this assumption does not always hold true, as mentioned above. Other approaches to increasing HDR have included using a ribonucleoprotein (RNP) complex of Cas9 and sgRNAs, synchronizing cell's cycles, "cold shocking" cells after transfection and nucleofection, and adding small molecules that enhance or inhibit HDR and NHEJ, respectively. Combining these approaches in the same experiment has been reported to yield scarless editing with high efficiency, but this was tested in only one cell line ${ }^{19}$.

\section{Limitations of current scarless editing methods}

Several methods have also been developed using the standard CRISPR/Cas9 editing tools to facilitate scarless editing ${ }^{20}$. However, these methods have one or more of the following limitations: (i) low editing efficiency (1-5\%); (ii) laborious and/or expensive clone screening; (iii) dependence on a PAM site near the target base; (iv) requirement of a specific genotype in the starting cell line (when editing the PAM site); (v) inconsistent experimental procedures for edited and unedited clones in experiments comparing isogenic clones.

In recent years, alternative Cas9-based editors were developed that allow for scarless editing without producing DSBs, such as base editors ${ }^{21 ; 22}$ and prime editors ${ }^{23}$. While both tools demonstrate high on-target editing efficiency, each has significant limitations.

Base editors can only perform transition mutations $(C \rightarrow T$ or $A \rightarrow G)$ and alter all vulnerable bases within a target window of 1-6 bp. Additionally, cytosine base editors show evidence of widespread and unpredictable off-target effects ${ }^{24}$. Prime editors can create a wider array of mutations but may also result in unintended integration of pegRNA sequences into the genome ${ }^{23}$. They have also almost exclusively been tested in neoplastic cells and their off-target effects are not well characterized.

To overcome these limitations, we developed CRISPR Deletion and Reinsertion (CRISPR Del/Rei, Fig. 2): a simple, two-step method that scarlessly edits iPSCs at high efficiency. This method is designed to be as user-friendly as possible and utilizes accessible, standard reagents with no specialized equipment.

\section{RESULTS}

\section{CRISPR Del/Rei overview and workflow}

CRISPR Del/Rei consists of two rounds of genome editing. Step 1 (Fig. 2a), creates a deletion of $\sim 45$ - 
$110 \mathrm{bp}$. This deletion is mediated by two sgRNAs that guide Cas 9 to remove the sequence between them. This sequence includes the target base and parts of the protospacers but, importantly, spares at least one of the two PAM sites. Once NHEJ repairs the deletion (Fig. 2b), the protospacers are disrupted, preventing repeated Cas 9 cleavage and leading to high deletion efficiency ( 15-100\%, Fig. 4, Supplementary Fig. 3).

In Step 2, the deleted sequence containing the desired edit is reinserted (Fig. 2c). The PAM site(s) preserved in Step 1 allows the sequence spanning the deletion junction to be used as the protospacer for a new sgRNA, which we term "synthetic" sgRNA or synsgRNA. The syn-sgRNA and a single stranded oligodeoxynucleotide (sSODN) template containing any allele of the target base facilitate the reinsertion of the desired sequences by HDR. Because the syn-sgRNA protospacer spans the deletion junction, the reinsertion pushes this sequence apart, preventing further synsgRNA binding and Cas9 cleavage, and increasing HDR efficiency (up to $20 \%$, Fig. 4, Supplementary Fig. 3).

The workflow for CRISPR Del/Rei (Fig. 3) is optimized for ease of use and high efficiency in cells that are difficult to transfect. The size of the deletion created in Step 1 (Fig. 2a) is flexible and can be adjusted to accommodate available PAM sites. Once designed, sgRNAs and syn-sgRNAs are cloned with a streamlined protocol 25 into the all-in-one vectors pDG459 or pX459, respectively. Using these single,

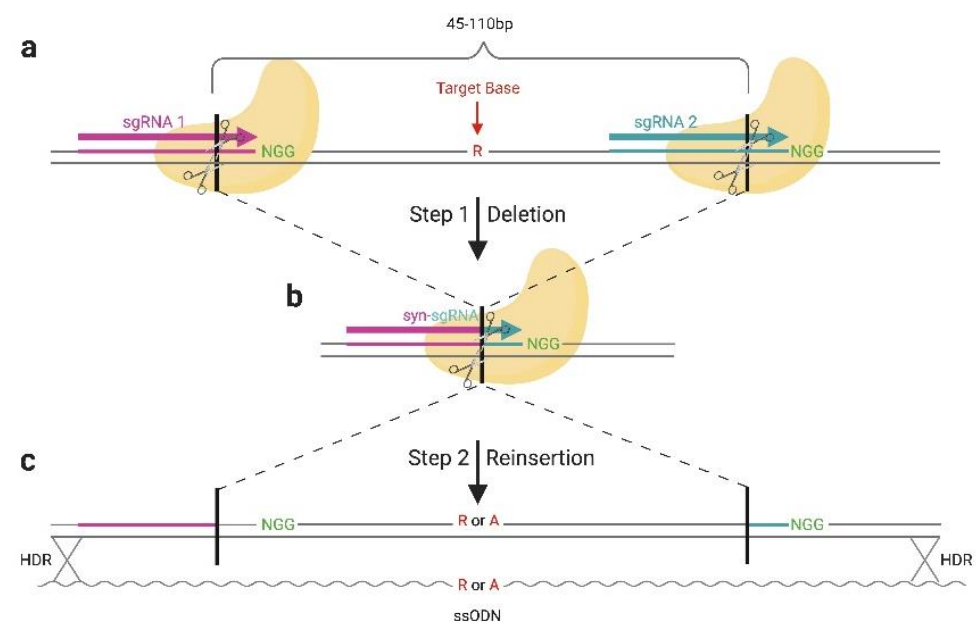

Figure 2 | CRISPR Del/Rei overview. (a) In Step 1, two sgRNAs (pink and blue) mediate a deletion containing the target base (red, "R" for reference allele) and portions of the protospacers (pink and blue). Importantly, one PAM site (green) is left external to the deletion. (b) Once the deletion is made, the protospacers are destroyed which prevents further Cas9 recruitment and cleavage, resulting in high editing efficiency. In Step 2, the sequence spanning the deletion junction (black vertical line) is used as a protospacer for a synthetic sgRNA (syn-sgRNA, pink and blue). The syn-sgRNA coupled with the spared PAM site mediates reinsertion of the deleted sequence, including any desired allele of the target base ("A" for alternate allele). (c) Once the sequence is reinserted by HDR, the syn-sgRNA protospacer is destroyed, again preventing additional Cas9 cleavage and yielding high editing efficiency.

compact plasmids not only reduces cytotoxicity by limiting the amount of DNA needed for transfection; but also eliminates the need for synchronous delivery of multiple vectors to each cell to ensure receival of all components needed for editing. Transfection is done

Figure 3| CRISPR Del/Rei Workflow. Editing is completed across two transfections using accessible reagents and an easy PCR-based screening method. The whole process takes about two months.

\section{Step1 - Deletion}

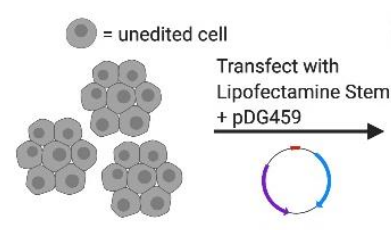

Day $1-2$
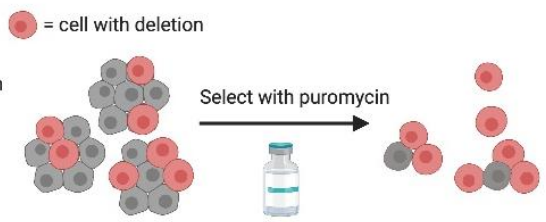

Day 3-4

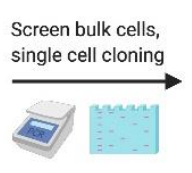

Day $4-14$

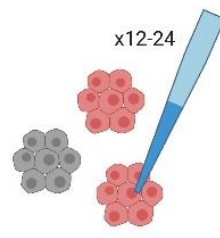

Day $15-25$

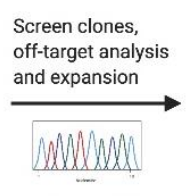

Day 15-25
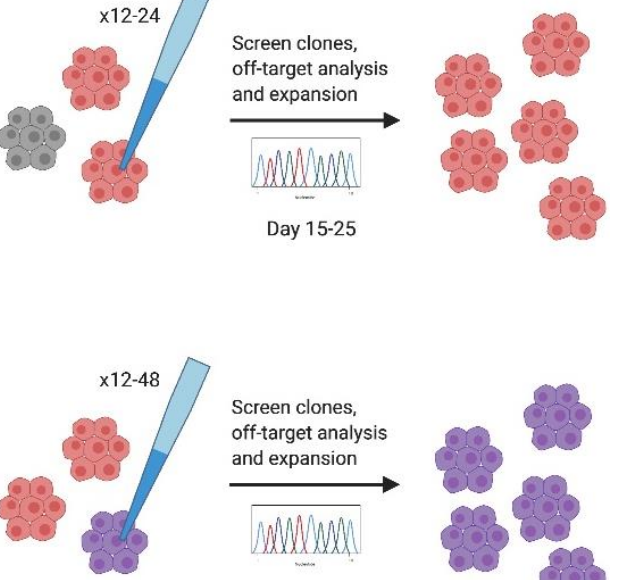

Day $30-40$

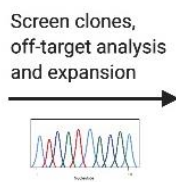

Day 41-55

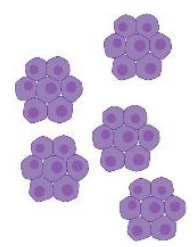

Total Time $=\sim 55$ days 
with Lipofectamine Stem, which requires limited handson time and is less stressful for cells than comparable protocols like electroporation. Transfected cells undergo positive selection with the antibiotic puromycin, which compared to other selection methods like cell sorting is much cheaper and has a reduced risk of cellular stress, death and contamination. After selection, editing efficiency is first determined in bulk populations of cells from each transfected well. After this the well with the desired efficiency can be sparse plated for single-cell cloning. The bulk transfected cells and individual clones are easily screened for the edit using simple PCR amplification and gel electrophoresis, with no restriction enzyme digest required like in other protocols ${ }^{26 ; 27}$. For Step 1, the edit can be visualized on a gel by the presence of a smaller deleted band (Fig. 4a-b, Supplementary Fig. 3). For Step 2, a larger fragment will be present when the reinsertion is successful (Fig. 4c-d, Supplementary Fig. 4). The edits can be confirmed by Sanger sequencing using the same primers used for PCR, after which positive clones are expanded and screened for off-target edits. Only one deletion clone is required to complete Step 1, and therefore off-target effects can be mitigated by selecting a clone free from extraneous editing. For Step 2, depending on the user's preference, alleles can be reinserted sequentially or in parallel via separate transfections; or simultaneously in a single transfection with an equimolar mix of ssODNs containing each allele. The whole process takes about 8 weeks.

\section{Step 1: Homozygous deletions at the loci TSNARE1, ATP2A2, NRGN, NRXN1, DPYSL2, and PKNOX2.}

We have successfully created 8 distinct deletions with high efficiencies in 6 schizophrenia-associated genomic regions in both coding and non-coding sequence across 7 different iPSC lines (Supplementary Tables 1, 2, and 5). Detailed methods and results for individual experiments are included in the supplementary material. Deletions ranged from 45-505 bp and were mediated by pairs of sgRNAs (Supplementary Table 1) cloned into the pDG459 plasmid using the protocol from

Figure 4 | Representative gels from the rs47766428 (ATP2A2) and rs4129585 (TSNARE1) experiments. (a) Screening of bulk transfected iPSCs following Step 1. Each lane represents a replicate transfected well from the same experiment. Deletions of rs4766428 (67bp) and rs4129585 (45bp) were successfully made with varying efficiencies ( 50-100\%). (b) Screening of single cell-derived deletion clones isolated from bulk transfected cells. Each lane represents a single clone. (c) Screening of bulk transfected iPSCs following Step 2. Each lane represents a replicate transfection well from the same experiment. rs4766428 (alternate and reference alleles) and rs4129585 (reference allele) were successfully reinserted with varying efficiencies ( 5-20\%) (d) Screening of single-cell-derived reinsertion clones isolated from bulk transfected cells. where each lane is a single clone. Alt= Alternative allele. Ref $=$ Reference allele. UE= unedited cells. Del= single deletion clone. Rei $=$ single reinsertion clone. Mix=mixed population of edited and unedited cells. Lanes separated by black line seen in $\boldsymbol{c}$ and $\boldsymbol{d}$ are from the same gel (cropped for conciseness), see Supplementary Fig 3.

a

\section{Bulk Deletions}

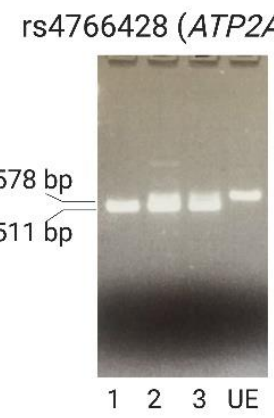

b

\section{Post Deletion Clones}

rs4766428 (ATP2A2) rs4129585 (TSNARE1)
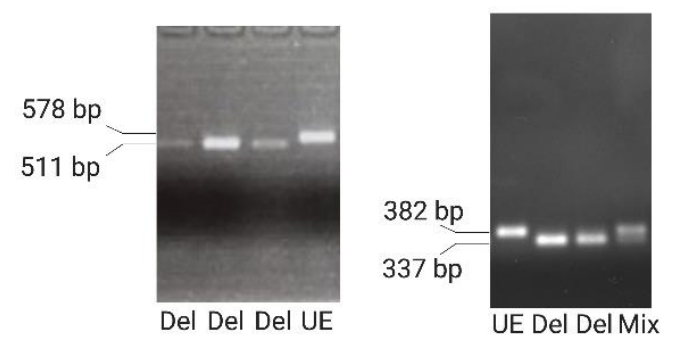

C

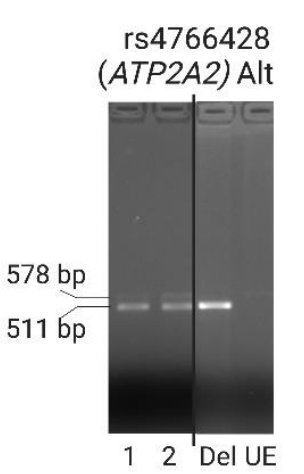

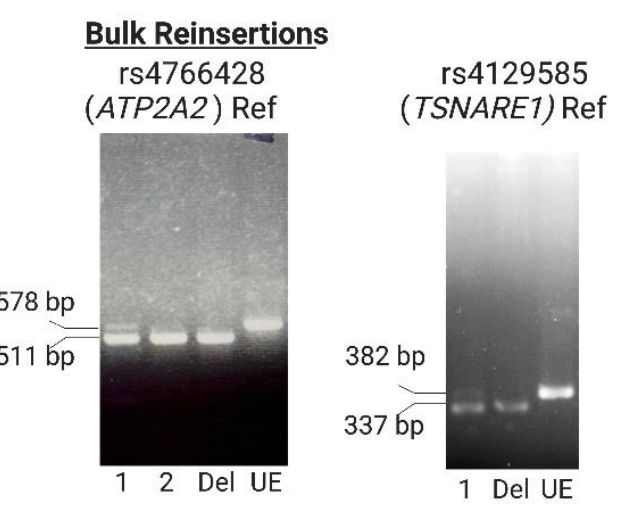

d

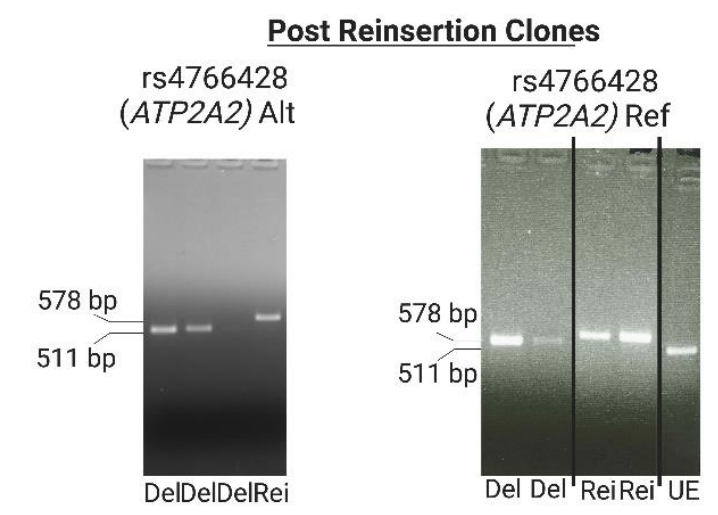


Adikusuma et al. ${ }^{25}$. iPSCs were transfected in replicate wells with pDG459 using Lipofectamine Stem. The transfection was followed by positive selection with puromycin (Supplementary Table 2). Genotyping of pooled cells from each transfected well showed deletion efficiencies between 15\% and 100\% (Fig. 4a, Supplementary Fig. 3), with some variation in editing efficiency between replicate wells (Supplementary Fig. 3). We isolated between 12 and 48 clones from the wells with the highest editing efficiencies by sparse plating and manual clone selection (Fig. 4a, Supplementary Fig. 3). On average, $\sim 15-100 \%$ of these clones were positive for deletion by gel electrophoresis. A total of 49 clones across all experiments were confirmed positive for the desired deletion by Sanger sequencing. In one case (ATP2A2 sgRNAs 4+2), the resulting deletion was slightly different from our design, which was likely due to the error-prone mechanism of NHEJ. This difference did not impede successful reinsertion as the PAM site near the deletion junction was retained, and therefore a synsgRNA could still be designed and utilized. This example highlights the need to confirm deletions by Sanger sequencing.

We performed Off-target analysis for edited clones using targeted Sanger sequencing. Candidate off-target sites were chosen by their likelihood of being a functional sequence. This selection process entailed prioritizing genomic regions with 4 or fewer mismatches to the protospacer and were located within exons or near open chromatin reported across all cell types by the Common Mind Consortium. Overall, across 5 experiments ( 30 clones, 43 candidate sites), we found off-target editing at only $2 / 628$ possible editing instances. Both clones were from the same experiment and the off-target edit was at the same site for both clones (Supplementary Table 3).

\section{Step 2: Homozygous reinsertion to complete desired edit at the loci TSNARE1 and ATP2A2}

Reinsertions were successfully performed at 2 of the loci confirmed to have deletions in Step 1 across 2 different iPSC lines (Supplementary Tables 1, 2, and 5). For each experiment, a single deletion clone, free from off-target editing was chosen for reinsertion. syngRNAs were designed to match the exact sequence of the clones' deletion site and to use the PAM site that was retained by design from Step 1. Syn-sgRNAs were cloned into $\mathrm{pX} 459$ using the same abbreviated protocol used for pDG459. ssODN repair templates were designed to be $160 \mathrm{bp}$ and have homology arms flanking the deletion site to facilitate HDR. One ssODN template was ordered for each allele (Supplementary Table 1). The confirmed deleted cells were again transfected using Lipofectamine Stem with pX459 and the ssODN template for each site and each allele
(Supplementary Table 2). The editing efficiencies for each locus were again estimated by PCR and gel electrophoresis. Genotyping of the bulk transfected cells revealed reinsertion efficiencies ranging from $\sim 5$ $20 \%$ (Fig. 4c). After single-cell cloning and manual selection of $24-48$ colonies, $\sim 7-40 \%$ of clones appeared positive by gel (Supplementary Fig. 4). Of the clones that were sequenced, $5 / 6(83 \%)$ harbored the correct, scarless edit. If a single round of sibselection ${ }^{28}$ was performed on the bulk transfected cells before single-cell cloning (Supplementary Fig. 4), $\sim 58-72 \%$ of clones appeared positive by gel (Supplementary Fig. 4), and 26/32 (81\%) of those sequenced harbored the correct scarless edit. Reinsertion clones were screened for off-target editing using the same prioritization pipeline as Step 1. No offtarget editing was detected (Supplementary Table 3).

\section{Creating Heterozygotes Cell Lines}

It is often desirable to create cell lines heterozygous for a deletion, a gene knock out or a variant of interest. CRISPR Del/Rei can be used to create heterozygous cell lines in two ways (Supplementary Fig. 1). The first method relies on the flexibility of placing the sgRNAs in the deletion step. One can design an sgRNA to target a protospacer harboring an existing heterozygous SNP in the targeted cell line. Because the sgRNA shares complete homology with only one of the alleles, only the complementary allele will be targeted and the resulting deletion is often heterozygous. We tested this in our rs4766428 (ATP2A2) deletion experiment using an iPSC line that contained a heterozygous, common SNP in the seed sequence of sgRNA4 (Supplementary Fig. 2a). Screening DNA from the bulk transfected cells showed a $\sim 50 \%$ deletion rate (Supplementary Fig. 2b), and after single-cell cloning $29 / 41$ clones $(71 \%)$ were heterozygous by gel electrophoresis (Supplementary Fig. 2c). The remaining clones were unedited (Supplementary Fig. 2c). This data demonstrates a highly efficient approach to make heterozygous deletions. A heterozygous deleted clone can then be used for a reinsertion where the syn-sgRNA will target only the deleted allele. The ability to create a deletion in one allele will allow the user to reinsert any alternative allele(s) to generate heterozygous clones; as well as reinsert the original allele to generate control clones.

The second method to design a heterozygous cell line is to create homozygous deletions in Step 1 but use an equimolar mix of HDR templates at Step 2 to reinsert all alleles simultaneously. This method is expected to produce clones that are $50 \%$ heterozygous and $25 \%$ homozygotes for each allele (in cases where there are two alleles). The transfection of cell lines with a mix of repair templates to generate heterozygotes has been previously described ${ }^{18}$. 


\section{DISCUSSION}

Here we demonstrate high efficiency scarless editing across multiple genomic regions and IPSC lines in $\sim 8$ weeks using our CRISPR Del/Rei strategy. This technique is user-friendly and relatively inexpensive and utilizes common laboratory reagents that are readily available. Additionally, CRISPR Del/Rei limits the number of clones that need to be manually selected; simplifies the screening process to PCR and gel electrophoresis; and it controls for experimental biases by using the exact same experimental procedure for all introduced alleles.

CRISPR Del/Rei has some limitations. Editing efficiency can vary based on factors such as inherent biological variability, differences in transfection efficiency across cell lines, and the characteristics of the target site. However, this holds true for most editing methods. An increased risk of off-target editing is another potential limitation of CRISPR Del/Rei as the technique uses three gRNAs (two sgRNAs and one syn-sgRNA). However, our data indicates that this risk is minimal (Supplementary Table 3). Furthermore, introducing large deletions (>110 bp) is not ideal for the reinsertion step because it would require the use of plasmids as HDR templates instead of ssODNs, which is known to be less efficient ${ }^{29}$. Our design requires the presence of 2 PAM sites, one that is external to the cut site, which may not apply to all sites in the genome. However, identifying such sites has not been problematic in our experiments so far.

Despite these limitations, CRISPR Del/Rei has many advantages over other gene editing methods, beyond the high editing efficiency. The ability to vary the size of the deletion allows for greater editing accessibility to genomic regions that are challenging to target due to factors like scarcity of PAM sites and/or repetitive regions that make sgRNA design difficult. The transfection protocol is flexible and customizable. Lipofectamine Stem and puromycin were chosen for the transfection and the selection steps, respectively, because they are easy to use, accessible, and inexpensive. Other transfection and selection methods such as electroporation and fluorescently-activated cell sorting (FACS) or magnetically-activated cell sorting (MACS) can also be used, but may alter the current experimental timeline. CRISPR Del/Rei was designed and applied in iPSCs, which are extremely useful to generate cellular models, but are difficult to transfect and edit. This novel approach is expected to have even higher efficiency in commonly used tumor-derived cell lines that are often more amenable to editing than iPSCs.

CRISPR Del/Rei is also a versatile technique with respect to the type and the location of genetic changes. As demonstrated, CRISPR Del/Rei can effectively create deletions, insertions, and single base pair changes. The strategy also has potential to make other complex modifications since the insertion step enables the introduction of any sequence. Here, CRISPR Del/Rei was used for scarless editing of noncoding sequences but it can also be applied in coding sequences instead of CRISPR-blocking mutations to ensure the integrity of protein production and function. In cases of coding mutations, using CRISPR Del/Rei may be a necessity, if CRISPR-blocking mutations are not compatible with synonymity. Additionally, because CRISPR Del/Rei's first step is a deletion, a knock-out of the variant(s) or gene(s) of interest is acquired, which can be useful to study in addition to the edited clones. An example of this are the deletions we made in DPYSL2 and NRXN1, which were placed in early exons to facilitate knock-out. CRISPR Del/Rei also enables using heterozygous lines of an allele of interest to generate homozygotes, which eliminates the need to start with a cell line of a particular genotype. Experimental variability is controlled because the unedited controls undergo the same experimental procedures of transfection, and genome editing as the edited clones. This novel strategy for applying CRISPR/Cas9 technology is of advantage for studying disease-associated variants in stem cells, especially for the generation of isogenic lines. Here we reported multiple examples on the efficiency of CRISPR Del/Rei. Our laboratory continues to work on seamless editing, and we expect to be reporting on additional supporting results in the near future.

\section{METHODS: \\ Cell lines and cell culture}

iPSC lines were obtained from the National Institute of Mental Health (NIMH/RUCDR) stem cell repository (Supplementary Table 5, https://www.nimhgenetics.org/). Before to cell seeding, tissue culture plates were coated with $5 \mu \mathrm{g} / \mathrm{mL}$ laminin (BioLamina \#LN521) and incubated at $37^{\circ} \mathrm{C}$ for at least two hours. Once seeded, the iPSCs were maintained in a feeder-free culture in StemFlex media with supplement (Gibco \#A3349401) at $37^{\circ} \mathrm{C}$ with $5 \% \mathrm{CO}_{2}$. The media was supplemented with 10 $\mu \mathrm{M}$ ROCK inhibitor (Y-27632 dihydrochloride, Tocris \#1254) on days of thawing, passaging, and transfection. Cells were passaged when $\sim 70-80 \%$ confluent every $\sim 4-5$ days with $1 \mathrm{X}$ Phosphate-buffered saline (PBS) and Accutase (MilliporeSigma \#A6964). Cells were frozen in StemFlex media with $10 \%$ Dimethylsulfoxide (DMSO). Genotyping of CRISPR targets was done by PCR and Sanger sequencing using primers (25nmole standard desalted, IDT) listed in Supplementary Table 1 and confirmed by SNP array (see below). 


\section{Quality Control and authentication of IPSC lines} All iPSC lines were genotyped at the Johns Hopkins University School of Medicine genetics core resource facility on the Illumina Global Screening Array-24 v2.0 to exclude major chromosomal abnormalities and confirm their identity.

\section{Design of sgRNAs for deletions (Step 1)}

The CRISPOR tool (http://crispor.tefor.net/) was used to identify candidate sgRNAs for each target. sgRNAs were prioritized based on three criteria: location, specificity score, and efficiency score. The location of the sgRNA pair and the resulting deletion they would mediate (Fig. 2) was critical for several reasons. First, the deletion was designed such that the target base(s) was near the middle to ensure its removal. Second, the deletion needed to be $\sim 45-110 \mathrm{bp}$, which is large enough to easily detect by gel electrophoresis, but small enough to accommodate use of an ssODN repair template. Third, at least one of the two PAM sites needed to be external to the deletion to ensure that one was left intact for use by a syn-sgRNA for reinsertion. Fourth, sgRNAs were placed in consideration of any local heterozygous variation. If clones that were homozygous for the target base(s) were desired, sgRNAs were placed to avoid including any heterozygous variants in the deletion. This placement avoided the need to restore such heterozygosity during reinsertion. Fifth, if heterozygous clones were desired and Method 1 for creating heterozygotes was being used (Supplementary Fig. 1) one sgRNA was placed so that a heterozygous SNP was in the seed sequence of the protospacer (Supplementary Fig. 2). Finally, in cases where gene knock-out was desired (DPYSL2 and NRXN1) the sgRNAs were placed so the deletion removed known functional domains (i.e. transcription start sites and/or start codons).

If there were still multiple sgRNA options available after considering the necessary location restrictions, those with high specificity and efficiency scores were preferred. For each variant, multiple sgRNAs were selected and tested as different pairs. The sgRNAs that successfully created deletions and the coordinates of the resulting deletions are listed in Supplementary Table 1.

\section{Design of syn-sgRNAs and ssODNs for reinsertions (Step 2)}

Syn-sgRNAs were designed to match the exact sequence of the corresponding deletion from Step 1. This was determined by Sanger sequencing. They were positioned over the junction of the deletion such that they utilized one of the remaining PAM sites from the sgRNAs (Fig. 2). Before ordering the syn-sgRNA, the sequences were queried against the human genome using NCBI BLASTn
(https://blast.ncbi.nlm.nih.gov/Blast.cgi) to confirm the absence of identical genomic sites.

The 160bp ssODNs (4 nmol PAGE-purified, IDT) were designed to have homology arms of at least 45bp flanking the deletion from Step 1. Sequences for syn-sgRNAs and ssODN repair templates are listed in Supplementary Table 1.

\section{Cloning}

sgRNAs were purchased as single-stranded DNA oligos (25nmoles standard desalted, IDT) with BbsI sticky ends and cloned in pairs into the pDG459 plasmid (Addgene \#10090) for the deletion step using a one-step cloning reaction as described by the Thomas lab ${ }^{25}$ The same protocol with the minor modification of replacing the second oligo with water during ligation was used to clone the syn-sgRNAs into pX459 (Addgene \#62988). A cloning mixture volume of $5 \mu \mathrm{L}$ or $10 \mu \mathrm{L}$ was then transformed into $25 \mu \mathrm{L}$ or $50 \mu \mathrm{L}$, respectively, of One Shot TOP10 Chemically Competent E.Coli (ThermoFisher \#C404003) following the manufacturer's protocol. Transformed bacteria were plated on carbenicillin or ampicillin LB agar plates and incubated at $37^{\circ} \mathrm{C}$ overnight. Individual colonies were picked into $3-5 \mathrm{~mL}$ of LB broth and cultured at $37^{\circ} \mathrm{C}$ overnight. Isolation of the plasmid DNA from bacterial clones was conducted using Zymo or Qiagen mini prep and maxi prep kits (Qiagen \#27104, Zymo \#D4208T)

\section{Transfection and selection}

Transfection and selection parameters differed slightly between experiments due to variability in viability, transfection and editing efficiencies across iPSC lines and genomic loci. The details for each experiment are listed in Supplementary Table 2. To summarize, cells were plated 24-48 hours before transfection at densities between 40k-60k cells/well on 24-well plates coated with laminin. The cells were transfected with 500ng of pDG459 (Step 1) or pX459 and ssODN templates at a 1:20 molar ratio using $2 \mu \mathrm{L}$ of Lipofectamine Stem (ThermoFisher \#STEM00003) per well. We followed the published Lipofectamine Stem protocol (https://assets.thermofisher.com/TFSAssets/BID/manuals/transfection-psc-lipofectaminestem-stemflex-protocol.pdf), sometimes with alterations. Specifically, in some cases the cells were incubated in the DNA/Lipofectamine/Opti-MEM mixture for 5-7 hours instead of the recommended 4 hours because this increased the editing efficiency. Additionally, after the incubation period the DNA/Lipofectamine/Opti-MEM mixture for some cell lines was exchanged with StemFlex instead of being supplemented with StemFlex because this increased cell viability. Puromycin was added $\sim 24-48$ hours post transfection at optimal concentration for each cell line. 
The cells were left to recover for 3-7 days before passaging and screening for bulk editing efficiency.

\section{Screening and expansion of edited cells}

To screen for desired edits, genomic DNA from bulk cells of each transfected well was extracted using Quick Extract buffer (Lucigen \#QE0905T). following the protocol published by the manufacturer (https://www.lucigen.com/docs/manuals/MA150EQuickExtract-DNA-Solution.pdf). Briefly, 5-7 days after transfection media was collected from the transfected wells and spun down. Small pellets from floating/ dead cells were detected and the volume of Quick Extract solution volume added $(10-50 \mu \mathrm{L})$ was determined by how large the pellet was. This allowed for a quick screen of the bulk cells. If amplification using Quick Extract-derived DNA was not successful, then cells were expanded so a larger cell pellet could be obtained, and the Gentra Puregene Cell Kit was used for extraction instead (Qiagen \#158388). Amplicons 200-850bp flanking the target site (Supplementary Table 1) were amplified using $5 \mu \mathrm{L}$ (Quick Extract) or $1 \mu \mathrm{L}$ (Gentra Puregene) of extracted DNA and the AccuPrime Taq DNA Polymerase System (ThermoFisher \#12339016). A volume of $10-15 \mu \mathrm{L}$ of each PCR product was combined with $10 \mathrm{X}$ Bromophenol Blue loading dye and loaded into 1-2\% agarose gels and run at $80-120 \mathrm{~V}$ for $30-60$ minutes. The gel percentage, voltage and running time depended on the size of the amplicons and the expected deletion. Samples that appeared positive for editing by gel were sent for Sanger sequencing to determine the exact edited sequence.

Transfection wells with the highest editing efficiency underwent single-cell cloning, which consisted of sparsely plating 500 cells per well in 6-well plates ( $2 \mathrm{~mL}$ of media per well) and allowing colonies to develop over 7-10 days. Half of each colony was manually picked into a PCR tube in $\sim 10 \mu \mathrm{L}$ of media and the other half into one well of a 24- or 96-well culture plate. The half clones in PCR tubes were mixed with $10 \mu \mathrm{L}$ of Quick Extract buffer for DNA extraction, followed by PCR, gel electrophoresis, and Sanger sequencing to confirm genotype. Positive clones were then expanded for off-target analysis.

If reinsertion efficiency was lower than desired, sib-selection was performed to generate populations of cells enriched for the edit. This consisted of passaging bulk transfected cells into 96-well plates at 30 cells/well. Once the wells reached confluency, the media was harvested and spun down to obtain cell pellets. Pellets were mixed with $10-30 \mu \mathrm{L}$ of Quick Extract, and screening proceeded as described above. Sib-selection wells that showed enrichment for a reinsertion edit were then sparse plated for single-cell cloning, from which individual colonies were picked and screened.

\section{Off-target analysis}

All predicted off-target sites for each sgRNA and synsgRNA were obtained from the CRISPOR tool (http://crispor.tefor.net/). To narrow down the number of sites, we prioritized those that were most likely to be targeted (had 3 or less mismatches with our sgRNAs) and were most likely to be in functional sequence (in exons or in DNase1 hypersensitivity sites (DHS) from dorsolateral prefrontal cortex data obtained from ENCODE

(https://www.encodeproject.org/) DNase-I

hypersensitive site (DHS) peaks. If a small number of candidate off-target sites was identified we included additional sites with up to 4 mismatches and/or those within $100-500 \mathrm{bp}$ of a DHS. Individual clones with confirmed edits For Step 1 and Step 2 were tested for off-target edits by Sanger sequencing of prioritized offtarget sites and compared to the un-transfected line. All off-target sites and primers used are summarized in Supplementary Tables 3 and 4.

\section{ACKNOWLEDGEMENTS}

This work was supported in part by NIMH grants P50 $\mathrm{MH} 094268, \mathrm{R} 01 \mathrm{MH} 113215$ and RF1 MH122936 to DA. Figures were created with BioRender.com.

\section{REFERENCES}

1. Jinek, M., Chylinski, K., Fonfara, I., Hauer, M., Doudna, J.A., and Charpentier, E. (2012). A programmable dual-RNA-guided DNA endonuclease in adaptive bacterial immunity. Science 337, 816-821.

2. Cong, L., Ran, F.A., Cox, D., Lin, S., Barretto, R., Habib, N., Hsu, P.D., Wu, X., Jiang, W., Marraffini, L.A., et al. (2013). Multiplex genome engineering using CRISPR/Cas systems. Science 339, 819-823.

3. Ran, F.A., Hsu, P.D., Wright, J., Agarwala, V., Scott, D.A., and Zhang, F. (2013). Genome engineering using the CRISPR-Cas9 system. Nat Protoc 8, 2281-2308.

4. Featherstone, C., and Jackson, S.P. (1999). DNA double-strand break repair. Curr Biol 9, R759-761.

5. Yang, L., Guell, M., Byrne, S., Yang, J.L., De Los Angeles, A., Mali, P., Aach, J., Kim-Kiselak, C., Briggs, A.W., Rios, X., et al. (2013). Optimization of scarless human stem cell genome editing. Nucleic Acids Res 41, 9049-9061. 
6. Mali, P., Yang, L., Esvelt, K.M., Aach, J., Guell, M., DiCarlo, J.E., Norville, J.E., and Church, G.M. (2013). RNAguided human genome engineering via Cas9. Science 339, 823-826.

7. Byrne, S.M., Mali, P., and Church, G.M. (2014). Genome editing in human stem cells. Methods Enzymol 546, 119-138.

8. Miyaoka, Y., Berman, J.R., Cooper, S.B., Mayerl, S.J., Chan, A.H., Zhang, B., Karlin-Neumann, G.A., and Conklin, B.R. (2016). Systematic quantification of HDR and NHEJ reveals effects of locus, nuclease, and cell type on genome-editing. Sci Rep 6, 23549.

9. Yadegari, H., Biswas, A., Akhter, M.S., Driesen, J., Ivaskevicius, V., Marquardt, N., and Oldenburg, J. (2016). Intron retention resulting from a silent mutation in the VWF gene that structurally influences the 5 ' splice site. Blood 128, 2144-2152.

10. Duan, J., Wainwright, M.S., Comeron, J.M., Saitou, N., Sanders, A.R., Gelernter, J., and Gejman, P.V. (2003). Synonymous mutations in the human dopamine receptor D2 (DRD2) affect mRNA stability and synthesis of the receptor. Hum Mol Genet 12, 205-216.

11. Kimchi-Sarfaty, C., Oh, J.M., Kim, I.W., Sauna, Z.E., Calcagno, A.M., Ambudkar, S.V., and Gottesman, M.M. (2007). A "silent" polymorphism in the MDR1 gene changes substrate specificity. Science 315, 525-528.

12. Maurano, M.T., Humbert, R., Rynes, E., Thurman, R.E., Haugen, E., Wang, H., Reynolds, A.P., Sandstrom, R., Qu, H., Brody, J., et al. (2012). Systematic localization of common disease-associated variation in regulatory DNA. Science 337, 1190-1195.

13. Song, M., Yang, X., Ren, X., Maliskova, L., Li, B., Jones, I.R., Wang, C., Jacob, F., Wu, K., Traglia, M., et al. (2019). Mapping cis-regulatory chromatin contacts in neural cells links neuropsychiatric disorder risk variants to target genes. Nat Genet 51, 1252-1262.

14. Lettice, L.A., Heaney, S.J., Purdie, L.A., Li, L., de Beer, P., Oostra, B.A., Goode, D., Elgar, G., Hill, R.E., and de Graaff, E. (2003). A long-range Shh enhancer regulates expression in the developing limb and fin and is associated with preaxial polydactyly. Hum Mol Genet 12, 1725-1735.

15. Chatterjee, S., Kapoor, A., Akiyama, J.A., Auer, D.R., Lee, D., Gabriel, S., Berrios, C., Pennacchio, L.A., and Chakravarti, A. (2016). Enhancer Variants Synergistically Drive Dysfunction of a Gene Regulatory Network In Hirschsprung Disease. Cell 167, 355-368 e310.

16. Castel, S.E., Cervera, A., Mohammadi, P., Aguet, F., Reverter, F., Wolman, A., Guigo, R., lossifov, I., Vasileva, A., and Lappalainen, T. (2018). Modified penetrance of coding variants by cis-regulatory variation contributes to disease risk. Nat Genet 50, 1327-1334.

17. Mohammadi, P., Castel, S.E., Cummings, B.B., Einson, J., Sousa, C., Hoffman, P., Donkervoort, S., Jiang, Z., Mohassel, P., Foley, A.R., et al. (2019). Genetic regulatory variation in populations informs transcriptome analysis in rare disease. Science 366, 351-356.

18. Paquet, D., Kwart, D., Chen, A., Sproul, A., Jacob, S., Teo, S., Olsen, K.M., Gregg, A., Noggle, S., and Tessier-Lavigne, M. (2016). Efficient introduction of specific homozygous and heterozygous mutations using CRISPR/Cas9. Nature 533, 125-129.

19. Skarnes, W.C., Pellegrino, E., and McDonough, J.A. (2019). Improving homology-directed repair efficiency in human stem cells. Methods 164-165, 18-28.

20. Das, D., Feuer, K., Wahbeh, M., and Avramopoulos, D. (2020). Modeling Psychiatric Disorder Biology with Stem Cells. Curr Psychiatry Rep 22, 24.

21. Komor, A.C., Kim, Y.B., Packer, M.S., Zuris, J.A., and Liu, D.R. (2016). Programmable editing of a target base in genomic DNA without double-stranded DNA cleavage. Nature 533, 420-424.

22. Gaudelli, N.M., Komor, A.C., Rees, H.A., Packer, M.S., Badran, A.H., Bryson, D.I., and Liu, D.R. (2017). Programmable base editing of $A^{*} T$ to $G^{*} C$ in genomic DNA without DNA cleavage. Nature 551, 464-471.

23. Anzalone, A.V., Randolph, P.B., Davis, J.R., Sousa, A.A., Koblan, L.W., Levy, J.M., Chen, P.J., Wilson, C., Newby, G.A., Raguram, A., et al. (2019). Search-and-replace genome editing without double-strand breaks or donor DNA. Nature 576, 149-157.

24. Zuo, E., Sun, Y., Wei, W., Yuan, T., Ying, W., Sun, H., Yuan, L., Steinmetz, L.M., Li, Y., and Yang, H. (2019). Cytosine base editor generates substantial off-target single-nucleotide variants in mouse embryos. Science 364, 289-292.

25. Adikusuma, F., Pfitzner, C., and Thomas, P.Q. (2017). Versatile single-step-assembly CRISPR/Cas9 vectors for dual gRNA expression. PLoS One 12, e0187236.

26. Gupta, R.M., Hadaya, J., Trehan, A., Zekavat, S.M., Roselli, C., Klarin, D., Emdin, C.A., Hilvering, C.R.E., Bianchi, V., Mueller, C., et al. (2017). A Genetic Variant Associated with Five Vascular Diseases Is a Distal Regulator of Endothelin-1 Gene Expression. Cell 170, 522-533.e515. 
bioRxiv preprint doi: https://doi.org/10.1101/2021.01.18.427163; this version posted January 18, 2021. The copyright holder for this preprint (which was not certified by peer review) is the author/funder. All rights reserved. No reuse allowed without permission.

27. Kwart, D., Paquet, D., Teo, S., and Tessier-Lavigne, M. (2017). Precise and efficient scarless genome editing in stem cells using CORRECT. Nat Protoc 12, 329-354.

28. Miyaoka, Y., Chan, A.H., Judge, L.M., Yoo, J., Huang, M., Nguyen, T.D., Lizarraga, P.P., So, P.L., and Conklin, B.R. (2014). Isolation of single-base genome-edited human iPS cells without antibiotic selection. Nat Methods 11, 291-293.

29. Cong, L., and Zhang, F. (2015). Genome engineering using CRISPR-Cas9 system. Methods Mol Biol 1239, 197-217. 